\title{
Comparison of the effect of Collagen (amino acid) and Amnion (proteinase inhibitor) on the wound healing of soft tissues
}

\author{
Weko Adhiarto*, Sunardi Mangundjaja*, Makmuri Yusuf**, Bambang Pontjo*** \\ *Department of Oral and Maxillofacial Surgery Faculty of Dentistry Universitas Padjadjaran/ \\ Hasan Sadikin Hospital-Bandung, West Java, Indonesia \\ ** Department of Pathology Anatomy Faculty of Medicine Universitas Padjadjaran/ \\ Hasan Sadikin Hospital-Bandung, West Java, Indonesia \\ ${ }^{* * *}$ Department of Pathology Anatomy Faculty of Veterinary Medicine Bogor Agricultural Institute
}

(IPB)

\section{ABSTRACT}

The wound healing process of the soft tissue aims to accelerate the closing of the wound by recurring, restoring the function, and minimizing the scar tissue. This process has to occur in the surgery process to obtain better wound healing. The aim of this study was to know the stimulation effect of wound dressing to accelerate the wound healing process of the soft tissue using Collagen (amino acid) and Amnion (proteinase inhibitors). This true experimental study was done to 36 mice (Sprague Dawley) that were divided into three different groups, two groups were the treatment groups and one group was the control group. Each group were adapted in Pathology Laboratory of Faculty of Veterinary Medicine Bogor Agricultural University. All of the mice were injured on the skin by incising the right side of the back for $2 \mathrm{~cm}$ in length and $4 \mathrm{~mm}$ in depth and than cleaned with $0.9 \% \mathrm{NaCL}$ solution. To the first group of treatment, the wound was applicated by Amnion, and the second group was applicated by Collagen. Each 4 mice of all group was termination by ether and necroption on the wounded tissue was conducted on day $3^{\text {rd }}, 7^{\text {th }}$ and $14^{\text {th }}$. Assessment for number of fibroblast and collagen synthesis on the wound of back tissue was conducted with the histological examination by painted using haematoxylin eosin. The results concluded that Collagen application was influencing the mean of fibroblast higher than Amnion, and the lowest was control with statistically significant. The mean of collagen used Collagen lower than Amnion, and the lowest was control with statistically significant. It could be concluded that collagen application was preeminent in increasing the productivity of the fibroblast, as well as in developing forms of the collagen synthesis compared with Amnion and control.

Key words: Wound healing of the soft tissue, Collagen (amino acid), Amnion (proteinase inhibitors).

\section{INTRODUCTION}

Soft tissue wound healing is a natural response to repair damaged tissue. Wound healing is also a complex interaction involving the cel- lular activity of skin repair, replacement of lost cells and restoration of both injured skin surfaces. Wound healing is a systematic process that consists of 3 phases: inflammation, proliferation, and maturation. Inflammatory phase is characterized

Correspondence author: Weko A, Department of Oral and Maxillofacial Surgery Faculty of Dentistry Universitas Padjadjaran Hasan Sadikin Hospital-Bandung Jl.Pasteur 38 Bandung, West Java-Indonesia, Tel./Fax.+6222-2041196/2036169 
by inflammatory cells and blood clots which are among the damaged tissue. Proliferation phase is marked by the epithelial, fibroplasia and angiogenesis process. Meanwhile, granulation tissue formation and the beginning of the unification of wound edge with the formation of collagen and protein molecules to increase the traction force (tensile strength) between the edges of the wounds are the phase of maturation. ${ }^{1}$

Soft tissue wound healing is a complex process, simultaneously and interact with each other when the injury occurred on a tissue that includes the physiology, biochemistry and morphology which are connected one to another. Wound healing process is also an ongoing series of events in the form of co-ordination of cellular activity, phagocytosis activity, chemotaxis activity, collagen synthesis and extra cellular matrix components synthesis. The process is essentially the same in all body tissues where the healing response begins shortly after a tissue undergoes an injury. ${ }^{2}$

Wound healing is an interrelated dynamic process that involves blood cells, mediator cells, extra cellular matrix, and parenchym cells. This process is profoundly influenced by several factors such as metabolic process and nutrition to accelerate wound healing process. ${ }^{3}$

The purpose of the soft tissue wound healing is to accelerate the process of wound closure and restore the function and minimize scar tissue formation. Besides, the most important aspects of the surgical procedure is to prepare for the wound healing process operating results going a good healing process. ${ }^{2}$ Wound healing process is a series of interrelated separate events and should be considered. Therefore, a surgeon needs to have an understanding of the wound healing process in order to obtain a wound healing results in anatomy form and the re-function, and also aesthetic value, so that a satisfying result can be obtained. ${ }^{4}$

Various materials for stimulating and accelerating soft tissue wound healing are used as a wound dressing, such as PDGF (platelet-derived growth factor), Collagen (amino acid) and Amnion (proteinase inhibitors) that basically restore the tissue's continuity and function and also a better cosmetic function. ${ }^{5}$

Collagen is amino acid matrix, while amnion (proteinase inhibitors) can accelerate wound healing of soft tissue, especially in the inflamma- tory phase and proliferation phase. In the inflammatory phase, the process is initiated by amino acids matrix with collagen that is exposed at the time of the injury that activates blood clotting process, intrinsically or extrinsically. This process initiates the process of inflammation. While the amnion inflammatory phase has a role in stimulating mast cells degranulation so it releases the histamine which accelerates the phase of acute inflammation. In the amnion proliferation phase, it stimulates fibroblasts to secrete collagen that can accelerate wound contraction and increase the tension strength of soft tissues' wound healing process. ${ }^{5,6}$

Based on those studies, the comparison of the effects of the use of collagen (amino acid) and amnion (proteinase inhibitors), to accelerate the process of wound healing of soft tissue will be observed.

\section{MATERIALS AND METHODS}

The subjects of this research were 36 mice (Spraque Dowley). The mice were divided into 3 groups (Group A, B and C). Group A and B were the treatment group and Group $C$ was a control. They had been adapted in the Pathology Laboratory of the Faculty of Veterinary of Bogor Agricultural Institute (IPB). The type of the research was pure experimental research with factorial design conducted to experiment animals, and the results were assessed using histopathologic examination.

The research has been done with made a wound to all of the mice on the skin soft tissue by incising the right side of the back for $2 \mathrm{~mm}$ length and $4 \mathrm{~mm}$ in depth. A wound was cleaned with $0.9 \% \mathrm{NaCl}$ solution, and then amnion were applied to group $A$, Collagen applied to group $B$, and Group C didn't have any treatment, except $\mathrm{NaCl}$ solution. On day 3, 4 mice from each group had the termination using ether and then necroption was carried out. All wound were taken and soaked in formalin solution. The assessment was performed with histological examination by having painted using haematoxylin eosin to see the number of fibroblasts and collagen. Preparation examination and assessment used the ocular microscope with 400 times' magnification. On day 7, 4 mice from group $A, B$ and $C$ had the some treatment as on day 3. Meanwhile, the rest of mice (4 mice) had 
the same treatment on day 14 .

To mention the number of fibroblast cells and the collagen fibers found, the unit used was the number of fibroblast cells per area of view (number of cells per LPB 400X), and the number of collagen fiber cells per area of view (number of fibers per LPB 400X). The number of fibroblast cells and collagen found were categorized as follows: 1-5/LPB (FSH) were low, 6-20/LPB (FSH) was moderate, 21-100/LPB (FSH) was high and> 100/ LPB (FSH) was massive. ${ }^{7}$

In order to found out the difference effect on collagen and amnion application to the three research variables, the statistical test was then performed, with ANAVA statistical in the factorial design of $3 \times 3$.

\section{RESULTS}

Before the statistical analysis conducted, for the type of numerical data, normality test was performed using the One Sample KolmogorovSmirnov Test to see the data distribution, normality test results could be seen in Table 1 . Normality test using the Kolmogorov Smirnov test showed that the variable number of fibroblasts and collagen was normally distributed ( $p>0.05$ ) so that the data was analyzed using a parametric test, ANAVA (Analysis variance).

The influence of provision collagen compared to Amnion on the number of fibroblasts can be seen in Table 2. Table 2 shows that the average number of fibroblasts in the treatment group with the application of collagen was 47.75 \pm 12.20 . The application of Amnion was lower than the application of collagen which was $43.92 \pm 12.56$ and the lowest was the control group, 30.50+8.48.

To find out the interaction between the treatment with the healing time of the number of fibroblast cells can be seen in Table 3. It can be seen that the use of Collagen and Amnion was significant. Similarly, the influence of time on the number of fibroblast was statistically significant. The interaction between treatments using collagen and amnion with wound healing time was not significant.

The influence of Collagen compared to Amnion on the number of collagen can be seen in Table 4. It can be seen that the mean of collagen number in application of Amnion was 32.13+5.94, in application of Collagen was 38.75+7.36 and the lowest was in the control group which was $21.50+7.43$.

To find out the interaction between treatments with healing time to the number of collagen can be seen in Table 5 . It is seen that the use of collagen and amnion was significant. The influence of time on the number of collagen was statistically non significant. Similarly, the interaction between treatments using collagen and amnion with wound healing time was not significant.

\section{DISCUSSION}

Wound healing is a complex biological process in tissue repair which include inflammation, re-epithelial, wound contraction, and collagen

Table 1. Variable normality test on the number of fibroblasts and collagen.

\begin{tabular}{ccc}
\hline \multirow{2}{*}{ Variable } & \multicolumn{2}{c}{ Normality test } \\
& P Value & Data distribution \\
\hline Number of fibroblast cells & 0.358 & Normal \\
Number of collagen cells & 0.161 & Normal \\
\hline
\end{tabular}

One sample Kolmogorov-Smirnov test

Table 2. The average number of fibroblasts cells to the effects of treatment and healing time.

\begin{tabular}{llccc}
\hline Treatment & Time & $\mathrm{n}$ & $\mathrm{x}$ & SD \\
\hline Amnion & Day 3 & 4 & 29.75 & 3.30 \\
& Day 7 & 4 & 56.25 & 8.96 \\
& Day 14 & 4 & 45.75 & 3.50 \\
Collagen & Total & 12 & 43.92 & 12.56 \\
& Day 3 & 4 & 37.25 & 5.74 \\
& Day 7 & 4 & 59.25 & 13.25 \\
& Day 14 & 4 & 46.75 & 3.50 \\
& Total & 12 & 47.75 & 12.20 \\
& Day 3 & 4 & 21.50 & 4.20 \\
& Day 7 & 4 & 39.75 & 1.71 \\
& Day 14 & 4 & 30.25 & 4.57 \\
& Total & 12 & 30.50 & 8.48 \\
\hline
\end{tabular}

Table 3. Analysis of variance of treatment effect on the healing time to the number of fibroblast cells.

\begin{tabular}{lcc}
\hline Variance source & F Calculation & P Value \\
\hline Treatment & 24.19 & $<0.001$ \\
Time & 36.49 & $<0.001$ \\
\hline Interactions & 0.58 & 0.681 \\
\hline ANAVA (analysis of variance). & &
\end{tabular}




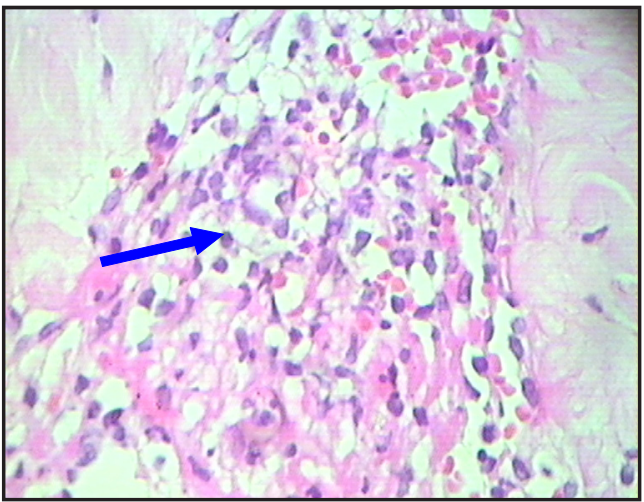

Figure 1. The photomicrograph result on day 3 without treatment (control). Blue arrow: fibroblast with medium category but still no collagen.

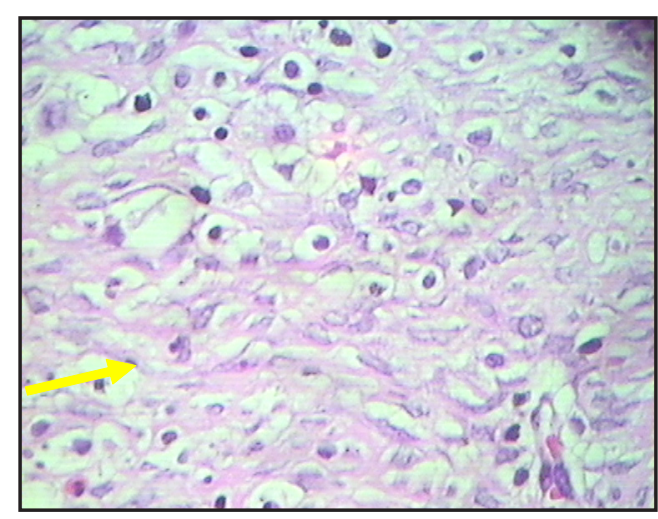

Figure 2. The result on day 7 (control), fibroblast with a high category and collagen (yellow arrow) with medium category.

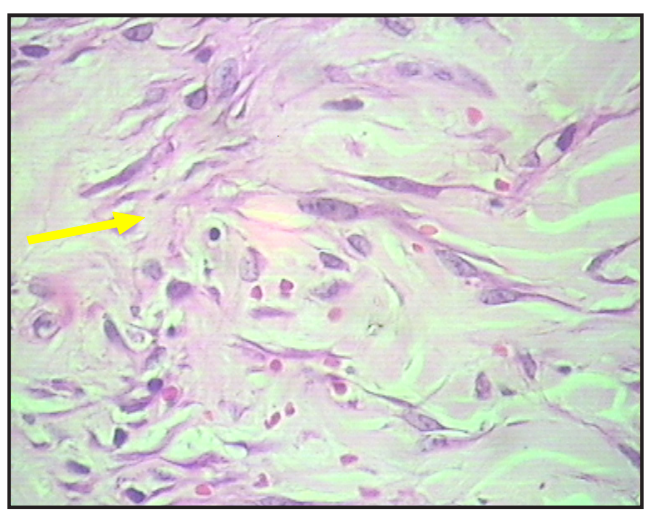

Figure 3. The result on day 14 (control), fibroblast and collagen (yellow arrow) in high category.

metabolism. ${ }^{8}$ At the beginning of wound occurrence, clot is formed, which is part of the inflammatory phase that is the early phase of wound healing process. Tissue injury will cause damage in blood vessels and will excrete blood components. Blood clots that are formed will maintain homeostasis and provide the extra cellular matrix

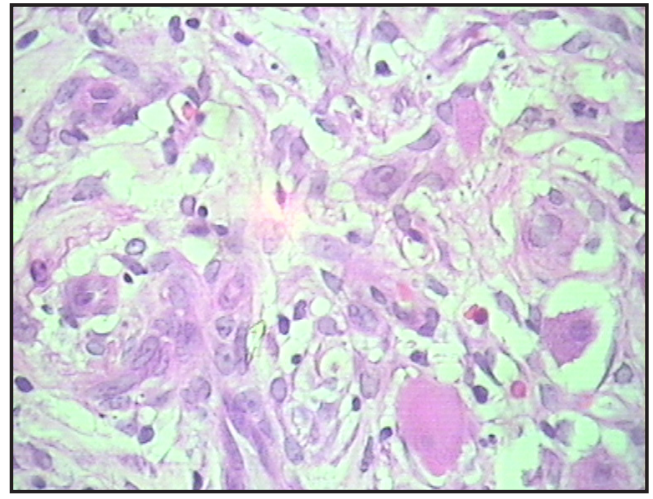

Figure 4. The result on day 3 with Amnion application, fibroblast with high category but still no collagen.

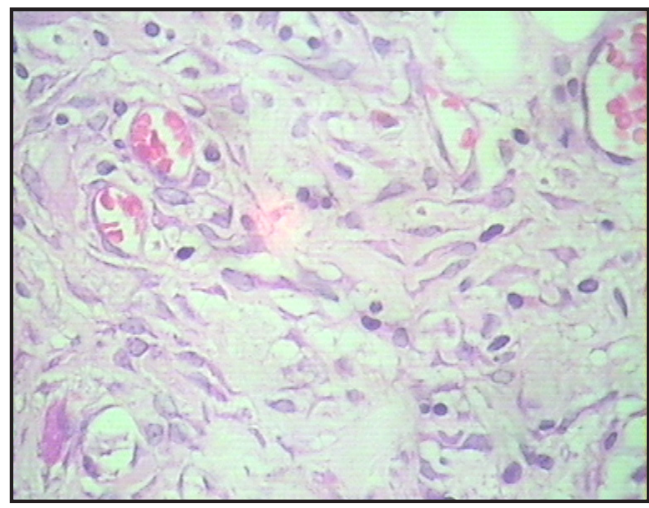

Figure 5. The result on day 7 with Amnion application, fibroblast and collagen with high category.

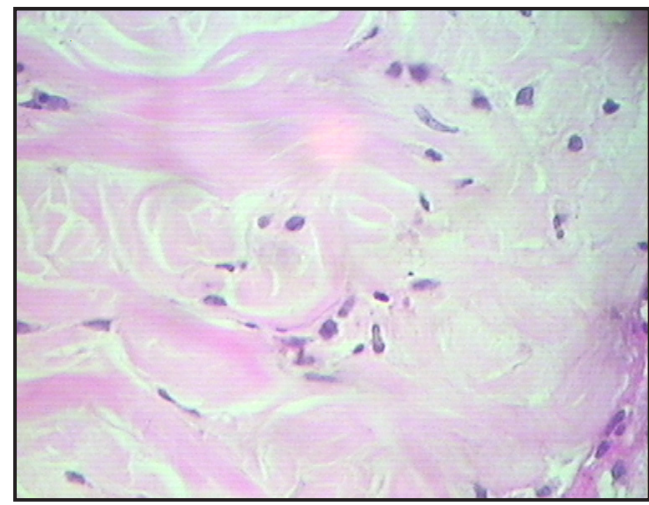

Figure 6. The result on day 14 with Amnion application, fibroblast and collagen with high categories.

for migration of cells such as neutrophil cells, endothelial cells, macrophages and fibroblast and collagen..$^{9,10}$

Factors that affect the speed of wound healing are shortening the inflammatory phase, the availability of sufficient oxygen, materials and energy required for cells regeneration. In the injured tissue, there will be metabolic disorders and tissue damages due to stress and hypoxia. To 


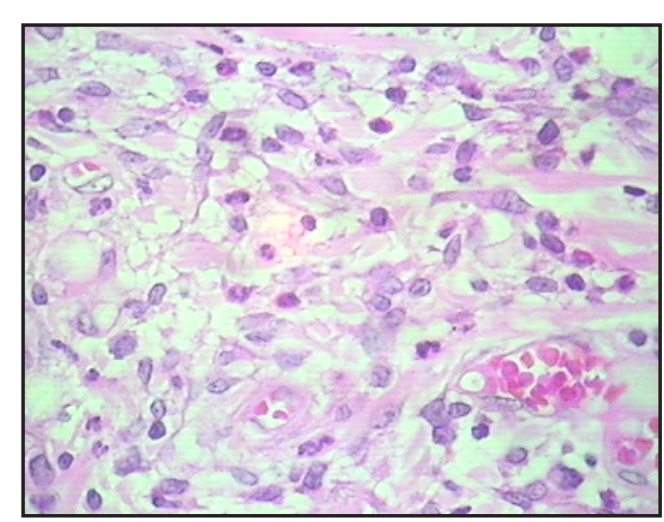

Figure 7. The result on day 3 with collagen application, fibroblast with high category but still no collagen.

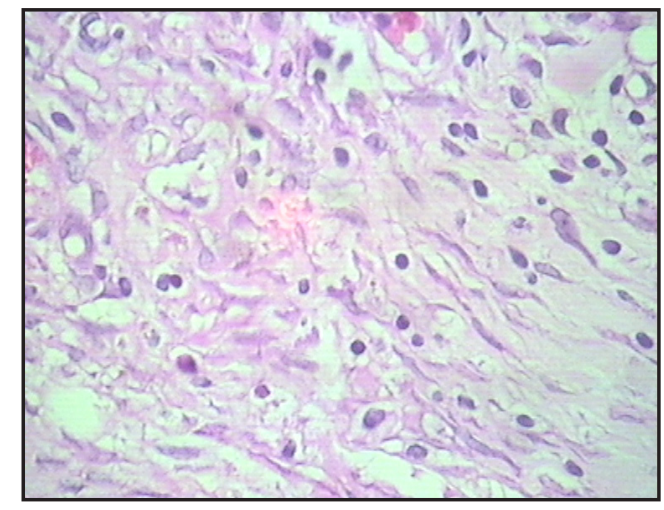

Figure 8. The result on day 7 with collagen application, fibroblast and collagen with high categories.

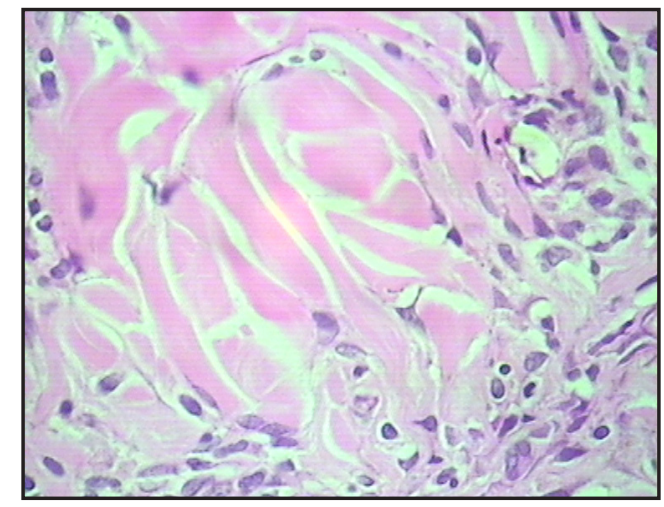

Figure 9. The result on day 14 with collagen application, fibroblast and collagen with high categories.

avoid the lengthening of the inflammation process as a result of infection, unrecognized things and the delay of wound treatment, the growth of material and energy, such as oxygen, amino acids, glucose, vitamins and the availability of oxygen to the tissue are needed..$^{11,12}$

Collagen is the amino acids matrix, while the Amnion is the material that can accelerate wound healing of soft tissue, especially in inflam-
Table 4. The average number of collagen to the effects of treatment and healing time.

\begin{tabular}{lllll}
\hline Treatment & Time & $\mathrm{n}$ & $\mathrm{x}$ & $\mathrm{SD}$ \\
\hline \multirow{3}{*}{ Amnion } & Day 7 & 4 & 29.50 & 6.03 \\
& Day 14 & 4 & 34.75 & 5.25 \\
& Total & 8 & 32.13 & 5.94 \\
Collagen & Day 7 & 4 & 40.00 & 10.80 \\
& Day 14 & 4 & 37.50 & 2.38 \\
& Total & 8 & 38.75 & 7.36 \\
\multirow{4}{*}{ Control } & Day 7 & 4 & 16.25 & 5.12 \\
& Day 14 & 4 & 26.75 & 5.38 \\
& Total & 8 & 21.50 & 7.43 \\
\hline
\end{tabular}

Table 5. Analysis of variance of treatment effect and healing time to the number of collagen.

\begin{tabular}{lcc}
\hline Variance source & F calculation & $P$ value \\
\hline Treatment & 15.06 & $<0.001$ \\
Time & 2.91 & 0.105 \\
\hline Interaction & 2.13 & 0.148 \\
\hline
\end{tabular}

*) ANAVA (analysis of variance).

matory phase and proliferation phase. The inflammatory phase is initiated by the amino acid matrix with collagen that are exposed during the wound occurrence which activates blood clotting process, intrinsically or extrinsically. This process initiates the process of inflammation. While the inflammatory phase of the amnion it has a role in stimulating mast cells release degranulation so it results histamine release which accelerates the phase of acute inflammation. In the proliferation phase of the amnion, it stimulates fibroblasts to secrete collagen that can accelerate wound contraction and increased tension strength of soft tissues' wound healing process. ${ }^{5,6}$

Collagen (amino acid) and Amnion are useful surgical materials that can accelerate wound healing process, restore tissue's continuity and function, accelerate wound contraction and increased tension forces on the wound healing process of soft tissue. ${ }^{5,6}$ Collagen and Amnion can also stimulate epithelial process and stimulate the formation of new blood cells and increase blood flow followed by the increase in collagen fiber tissue expansion so it can accelerate wound repair in soft tissue. ${ }^{13}$

According to the result that had been done using histopathology examination, on day 3 , in the treatment group there was high activation 
of fibroblast cells migration. It was influenced by the transforming of fibroblast growth factor that activated the fibroblast and stimulated epithelial acceleration in the healing process of soft tissues' wound. The process was initiated by the increase of blood flow towards the wound, initiated by thrombosis, followed by lymphocyte cells, neutrophil cells, keratinocytes, macrophage cells and fibroblast cells. These cells excreted cytokines and carried out their respective functions. The macrophage cells played an important role in the inflammation process for cleansing off died cells and damaged tissues by phagocytosis. While fibroblast cells play an important role in forming granulation tissue and tissue repair. ${ }^{2,10}$

In histopathology observation on the $3^{\text {rd }}$ day, there were differences in the average number of fibroblast in wound area between the treatment group and the control group. Statistically, in the treatment group the average number of treated fibroblast using amnion was 29.75 , the treatment using collagen averaged 37.25 . While the average number of fibroblasts in the control group was 21.5. This indicated that the fibroblast cells' migration rate in the treatment group was higher than the control group and in the treatment group using collagen was better than the treatment group using Amnion although it gave the effect of increasing the number of fibroblast, but the number of fibroblast produced was lower than in the treatment group using collagen.

Similarly, the number of fibroblast on day 7 and day 14 in the treatment group using Collagen showed better result, that was 59.25 fibroblast on day 7 and 46.75 fibroblasts on day 14, whereas in the control group on day 7 , there was only 39.75 fibroblasts and on day 1430.25 fibroblasts. It was due to the provision of collagen, growth factors contained in the collagen which regulates migration, proliferation, and cells differentiation to modulate the fibroblast cells that responded to growth factors, so the number of fibroblast was more than the control group that was not given any treatment. ${ }^{14,15}$

The influence of giving growth factors made the fibroblast secreting collagen which can accelerate wound contraction and increased the strength of the soft tissue in wound healing process. As the number of fibroblasts grew, the effects of growth factors (Collagen and Amnion) provision in the wound area, the next development would enhance and accelerate the process of collagen synthesis. ${ }^{15}$

During the first 3 days, the wound healing of epidermal cells that had been activated in the wound would secrete a large number of vascular endothelial cell growth factors which were the important factors for angiogenesis during the formation of granulation tissue on day 4 to day 7 . On the observation on day 7 and day 14 there was an increase in the fibroblast cells proliferation and the formation of collagen fibers. Statistically, at day 7 the average number of collagen in the control group was 16.25 , in the treatment group using Collagen reached 40 collagen fibers, in the group with treatment using Amnion reached 29.5 collagen fibers. The statistical test result a significant difference in the treatment group using Amnion and Collagen compared to the group with no treatment. This indicated that the application of Amnion and Collagen could improve the activation and proliferation of fibroblast cells and collagen synthesis in the group with treatment was higher than the control group.

The effect of growth factors giving (Collagen and Amnion) in wound area caused the increase in fibroblast cells' proliferation; high proliferation could affect the synthesis of collagen which increased as well. The synthesis of collagen on day 7 could be observed histopathologically with hematoxillin eosin staining and it could be found the new formation of collagen fibers. These collagen fibers were synthesized by fibroblast. ${ }^{16,17}$

On day 14 , there was a decline in the average number of collagen cells in the treatment group with collagen ( 37.5 collagen fibers) and treatment with amnion (34.75 collagen fibers) While in the control group there were 26.75 collagen fibers. This showed that between days 3 to day 14 there was collagen synthesis by fibroblast cells. The collagen was formed in accordance with the level of proliferation of fibroblast cells.

The main component of extra cellular matrix protein was collagen. In extra cellular matrix, there was an interaction between the integrin receptor fibroblast excreted by fibroblast with the extra cellular matrix itself. This interaction result the activation of fibroblast cells to make collagen synthesis..$^{10,18}$

The process of angiogenesis in wound heal- 
ing process is required, including for the metabolism of fibroblast cells. Angiogenesis is an event of forming new blood vessels that supply oxygen and nutrients needed in the process of cells' metabolism. ${ }^{15,17}$ Nutrients needs and oxygen supply are required for activation of proliferation and interaction with extra cellular matrix in the process of collagen fibers formation. The collagen fibers formed are required for tissue repair in remodeling phase. ${ }^{10,18}$

The provision of growth factors in wound healing of soft tissues proven to be able to increase high fibroblast cells migration activation with the use of Collagen on day 3 and proliferation activation of fibroblast cells was high on day 7 , also the formation of collagen fibers was high on day 7 and day 14 compared with the use of Amnion and control group.

\section{CONCLUSION}

There were differences in the use of collagen compared to amnion against the number of fibroblasts. There are differences in the use of collagen compared to Amnion against the amount of collagen. The influence of the use of collagen compared to amnion against the time of soft tissue wound healing was not statistically significant. The interaction of the use of collagen compared to amnion with the time of soft tissue wound healing was not statistically significant.

\section{SUGGESTIONS}

The use of collagen and amnion in wound healing can be given as a treatment option to accelerate soft tissue wound healing. The provision of collagen and amnion to post-surgery wound to accelerate soft tissue wound healing needs to be considered.

\section{REFERENCES}

1. Thomas R. Wound healing. Skin 2008. Available from: http://www.Li/wound heal.

2. Hupp JR. Wound repair. In: Peterson. Contemporary oral and maxillofacial surgery. $4^{\text {th }}$ ed. St.Louis: Mosby; 2003. p. 49-55.

3. Richard. Cutaneous wound healing. The New England J Med 1999;341(10).
4. Hom DB, Linzie BM, Huang TC. The healing effects of autologous platelet gel on acute human skin wounds. Arch Facial Plast Surg 2007;9(3):174-83.

5. Sclafani AP, Romo T, Ukrainsky G. Modulation of wound response and soft tissue ingrowth in synthetic and allogeneic implants with platelet concentrate. Arch Facial Plast Surg 2005;7(3):163-9.

6. Kim, Tseng. Transportation of preserved human amniotic membrane for surface reconstruction in severely damaged rabbit corneas. Archives of Optham Cornea 1995;15:473-84.

7. Ercan I. Prognostic significance of stromal eosinophilic infiltration in cancer of larynx. J Otolarygeal Head and Neck Surgery 2005;132:869-73.

8. Stadelmann WK, Digenis AG, Tobin GR. Physiology and healing dynamic of chronic cutaneous wounds. Am J Surg 1998; 176(Suppl 2A):26S-38S

9. Dujon GD. Wound healing. Sheffield: Dept of Plastic Surgery Northern General Hospital. 1996.

10. Andreasen JO. Textbook and color atlas of tooth impaction. $1^{\text {st }}$ ed. St. Louis: Mosby Year Book; 1997.

11. Ghoneimi B. Use of solcoseryl in minor burns. Ann Burns Fire Disast 1997.

12. Wai HT, Know T, Peter Pang. Facial scar management. In: Booth, Eppley and Schmelzeisen. Maxillofacial trauma and esthetic facial reconstruction. Spain: Churchill Livingstone; 2003.

13. Bose B. Burn wound dressing with human amniotic membrane. Ann R Coll Surg Eng 1999;61:444-7.

14. Robert F. Wound healing: an overview of acute, fibrotic and delayed healing. In: Frontiers Bioscience; 2004. p. 9,283-9.

15. Brown JA. The rule of hyaluronic acid on the wound healing process following extraction. J Oral Maxillof Surg 2004;243:124-7.

16. Robbins SL. Pathologic basic of disease. $4^{\text {th }}$ ed. Philadelphia: W.B. Saunders Co.; 1989.

17. Inkinen. Connective tissue formation in wound healing. Helsinki: University Central Hospital. 2003.

18. Romo T. Wound healing. Skin. 2003. Available from:http://www.wound heal/in rats. 